\title{
Adequacy of brain and spinal blood supply with antegrade cerebral perfusion in a rat model
}

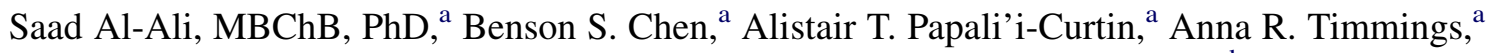 \\ Colleen Bergin, MBChB, Am Bd Cert Rad, ${ }^{a}$ Peter Raudkivi, MBChB, FRACS, ${ }^{\text {b }}$ and \\ Jeremy Cooper, MBChB, FFARACS, FANZCA ${ }^{\mathrm{c}}$
}

Objective: The purpose of this study was to examine whether different techniques used for antegrade cerebral perfusion could account for variation in the perfusion adequacy of the brain and spinal cord.

\begin{abstract}
Methods: Selected vessels were ligated in 30 rats, recreating a selection of approaches used in aortic arch surgery for patients undergoing circulatory arrest with antegrade cerebral perfusion. Filling of spinal and cerebral vessels was mapped after cannulation and perfusion with E20, gelatin/India ink, or buffered saline/India ink. Three clinical approaches were replicated: unilateral perfusion, bilateral perfusion, and bilateral perfusion with additional left subclavian artery perfusion. Filling of the spinal arteries via the common carotid arteries or the subclavian arteries alone was examined. Penetration of the marker was analyzed histologically.
\end{abstract}

\begin{abstract}
Results: The control experiments achieved maximal arterial filling of both brain and spinal cord at gross and microscopic levels. Unilateral and bilateral antegrade cerebral perfusion provided comprehensive arterial filling of all cerebral vessels with all vascular markers. In contrast, only bilateral antegrade cerebral perfusion provided complete spinal cord perfusion with all markers. Unilateral antegrade cerebral perfusion with a viscous marker resulted in significantly reduced spinal cord arterial filling. Examination of the relative importance of either both common carotid arteries alone or both subclavian arteries alone, in terms of their adequacy of subsequent arterial filling of the spinal cord, showed severe impairment of spinal cord perfusion with either technique. Thus perfusion of both common carotid arteries resulted in only the proximal $30 \%$ of the spinal cord arteries being filled, whereas perfusion of both subclavian arteries resulted in only the proximal $40 \%$ of the spinal cord arteries being filled.

Conclusions: Approaches to antegrade cerebral perfusion using the brachiocephalic and left common carotid arteries together gave good perfusion of both the brain and the spinal cord. Brachiocephalic perfusion alone gave good cerebral perfusion but showed some significant limitation in spinal cord perfusion with one vascular marker. Complete spinal cord perfusion with all markers under conditions of antegrade cerebral perfusion required some contribution from both the carotid system and the subclavian system together. Selected perfusion of either system alone was very inadequate for spinal cord perfusion. (J Thorac Cardiovasc Surg 2011;141:1070-6)
\end{abstract}

Antegrade cerebral perfusion (ACP) is a widely used method of cerebral protection performed during surgical repair of the aortic arch under conditions of hypothermia and visceral circulatory arrest. ${ }^{1,2}$ The blood supply to the brain and spinal cord during circulatory arrest is maintained with ACP via cannulation and perfusion of selected arteries arising from the aortic arch. Several methods of cerebral

From the Departments of Anatomy with Radiology, ${ }^{\text {a }}$ FHMS, University of Auckland, Cardiothoracic Surgery, Auckland City Hospital, ${ }^{\mathrm{b}}$ and Department of Cardiothoracic and ORL Anaesthesia, ${ }^{c}$ Auckland City Hospital, Auckland, New Zealand.

Funding for this project was provided by the Green Lane Fund (07/514056-9135/ 3620460) and by the University of Auckland Research Options Fund.

Disclosures: Authors have nothing to disclose with regard to commercial support.

Received for publication Dec 10, 2009; revisions received March 22, 2010; accepted for publication Jan 19, 2011.

Address for reprints: Saad Al-Ali, MBChB, PhD, Department of Anatomy with Radiology, University of Auckland, Private Bag 92019, Auckland, New Zealand (E-mail: s.al-ali@auckland.ac.nz).

$0022-5223 / \$ 36.00$

Copyright (C) 2011 by The American Association for Thoracic Surgery doi:10.1016/j.jtcvs.2011.01.027 protection are in clinical use, including metabolic suppression with anesthetic agents, ACP, hypothermic circulatory arrest, and retrograde cerebral perfusion. ${ }^{3}$ All have been directed at reducing operative mortality for aortic arch surgery and reducing the perioperative incidence of temporary and permanent neurologic deficits.

One of the devastating potential deficits is paraplegia, which results from spinal cord ischemia. The incidence of paraplegia under conditions of circulatory arrest and hypothermia is most often studied in surgery of the thoracic and thoracoabdominal aorta, where a rate of $2.7 \%$ has been described. ${ }^{4}$

Although ACP is a widely established technique, vessel selection for cannulation and perfusion varies in clinical practice. $^{3,5-9}$ Two ACP approaches are commonly used: unilateral ACP, performed with arterial cannulation of the brachiocephalic artery or some of its branches, and bilateral ACP, performed by cannulating both the brachiocephalic and left common carotid arteries. ${ }^{6,710,11}$ An adjunct to the latter is cannulation and perfusion of the left subclavian artery. ${ }^{3}$ 


\section{Abbreviation and Acronym \\ $\mathrm{ACP}=$ antegrade cerebral perfusion}

Neurologic deficits associated with each of these methods vary, but some clinical reports suggest that bilateral ACP is better than unilateral ACP with lower associated hospital mortality. ${ }^{12}$ A recent review comparing unilateral and bilateral perfusion found that rates of neurologic deficit for both were below $5 \% .{ }^{9}$ However, the period of safe operative time allowed with bilateral perfusion was significantly longer. Unilateral ACP permitted cerebral protection from 30 to 50 minutes with an acceptably low rate of cerebrovascular accidents, whereas bilateral ACP permitted 86 to 164 minutes of cerebral protection with a similar low rate of cerebrovascular accidents. ${ }^{9}$

The purpose of this study was to examine whether different techniques used for ACP could account for variation in susceptibility to neurologic deficits. We used a rat model to study perfusion adequacy in the brain and spinal cord, recreating a selection of approaches used in aortic arch surgery for patients undergoing circulatory arrest with ACP. Filling of spinal and cerebral vessels was mapped after cannulation and perfusion in 3 approaches to ACP: (1) unilateral perfusion, (2) bilateral perfusion, and (3) bilateral perfusion with additional left subclavian artery perfusion.

Blood flow to the spinal cord through the internal carotid and vertebral circulations was also studied by cannulation and perfusion of the common carotid arteries and subclavian arteries separately. The filling of spinal cord arteries and their penetrating branches was mapped by crosssectional histologic examination by which we studied which vessels, when selectively cannulated, achieved the greatest extent of filling in these arteries.

Previous studies have reported close similarities between the rat and human spinal cord vasculature. ${ }^{13,14}$ Therefore, the rat is well suited as an experimental model for investigating the vascular supply to the spinal arteries.

\section{MATERIALS AND METHODS}

This study was approved by the Animal Ethics Committee, University of Auckland, and strictly followed animal welfare guidelines (AEC/09/ 2005/T412).

\section{General Procedures}

The vasculature of the rat spinal cord was investigated by perfusion of specific vessels with vascular markers, either colloidal carbon 50\% (India ink, Pebeo) mixed with buffered saline or gelatin $2 \%$ to $5 \%,{ }^{15}$ or an epoxy resin mixture (E20; Biodur Products, Heidelberg, Germany). For the E20 marker a ratio of 100:45:10 of E20:E2 methyl ethyl ketone mixture was used. The markers were delivered into the vessels by 2 different techniques. The colloidal carbon/saline mixture was delivered by a hydrostatic pressure system (at a height of $1 \mathrm{~m}$ ), whereas the colloidal carbon/gelatin mixture and epoxy resin markers were manually delivered with a syringe (Table 1).
Thirty Wistar rats weighing 300 to $600 \mathrm{~g}$ were used. Anesthesia was established via intraperitoneal injection of $4 \%$ chloral hydrate $(1 \mathrm{~mL}$ per $100 \mathrm{~g}$ body weight), along with injections of heparin (anticoagulant) and $1 \%$ sodium nitrite (vasodilator). Once the rats were anesthetized, the abdominal cavity was opened with a midline incision, the thoracic cavity opened with bilateral midaxillary incisions, the diaphragm incised, and the anterior thoracic wall was reflected superiorly. A plastic cannula (outer diameter of $2 \mathrm{~mm}$ ) was inserted through the left ventricle and secured in the ascending aorta. Blood was washed from the vascular system by perfusing saline via this cannula. Then fixation was carried out by perfusion using a gravity-feed system with $4 \%$ buffered formalin solution. An incision in the right atrium enabled drainage. The time between the death of the rats (opening of the thoracic cavity) and the initiation of tissue fixation was less than 2 minutes. A standardized procedure was adopted to ensure that the tissue did not become edematous. Different vessels in the head and neck vasculature were then occluded with silk ligatures, while perfusion was performed with the vascular markers described earlier, to study the effect on brain and spinal cord perfusion.

Vascular supply was depicted by injecting either colloidal carbon or E20 via the cannula inserted through the left ventricle, with the core temperature of the rats at $20^{\circ} \mathrm{C}$. No other injection site was used.

The gelatin-based vascular markers were allowed to harden before dissection by maintaining the rats at $4^{\circ} \mathrm{C}$ for 2 days. Craniotomy and laminectomy were then performed to expose the brain and spinal cord in situ. The brain and spinal cord were photographed and then carefully removed en bloc. Filling of spinal vessels was examined and recorded with high-resolution digital photography using a Nikon 12.1 MP D2Xs camera (Nikon, Inc, Tokyo, Japan) and with a stereomicroscope (Leica MZ16) equipped with a Leica DFC290 digital camera (Leica, Solms, Germany). The length of the spinal cord from the brainstem to the conus medullaris was measured. The length of filling in the anterior and posterior spinal arteries was also measured and then converted into a percentage of the total length of the cord. For microscopic anatomy study, the cord was then sectioned horizontally at 3 levels (cervical, thoracic, and lumbar) and stained with methyl green stain to evaluate the penetration of the marker into the white and gray matter of the spinal cord.

\section{Selection of Arteries for Occlusion and Perfusion}

In this study 3 to 5 rats were allocated to each of groups I to V. These numbers were chosen to account for any perfusion failures, which is not uncommon in rat experiments. Furthermore, there are few anatomic variations in the vasculature of rats, unlike humans.

Five rats served as controls (group I). In these rats, no vessels were occluded. Rats were perfused with either gelatin/India ink, saline/India ink, or E20 (Figure 1, A).

The clinical scenario of unilateral ACP was replicated in 5 rats by occluding the left common carotid and left subclavian arteries at their origins and the descending aorta just distal to the origin of the left subclavian artery (group II). In this group, the vascular marker was directed into the brachiocephalic artery. Either gelatin/India ink or saline/India ink was used (Figure 1, $B$ ).

In 12 rats the clinical scenario of bilateral ACP was replicated by occluding the left subclavian artery at its origin and the descending aorta just distal to the origin of the left subclavian artery (group III). The vascular marker was directed into the brachiocephalic and left common carotid arteries. Either gelatin/India ink or saline/India ink was used (Figure 1, C). A total of 12 rats were used in this group as we attempted to optimize our perfusion methodology; therefore, more rats were used in this group.

Bilateral ACP with additional left subclavian artery perfusion was replicated in 3 rats by occluding the descending aorta just distal to the origin of the left subclavian artery (group IV). The gelatin/India ink marker was directed into the brachiocephalic, left common carotid, and left subclavian arteries (Figure 1,D). Perfusion with the saline/India ink marker was not performed.

In group $\mathrm{V}$, blood flow to the spinal cord alone via the internal carotid circulation was studied in 4 rats. In these rats, both subclavian arteries 
TABLE 1. Vascular markers used and the average extent of spinal arterial filling

\begin{tabular}{lcccccc}
\hline \multicolumn{1}{c}{$\begin{array}{c}\text { Vascular } \\
\text { marker }\end{array}$} & $\begin{array}{c}\text { Group I } \\
\text { (control) }\end{array}$ & $\begin{array}{c}\text { Group II } \\
\text { (unilateral ACP) }\end{array}$ & $\begin{array}{c}\text { Group III } \\
\text { (bilateral ACP) }\end{array}$ & $\begin{array}{c}\text { Group IV } \\
\text { (bilateral ACP+LSA) }\end{array}$ & $\begin{array}{c}\text { Group V } \\
\text { (CCA only) }\end{array}$ & $\begin{array}{c}\text { Group VI } \\
\text { (SCA only) }\end{array}$ \\
\hline E20 & $100 \%$ & $-*$ & $50 \%$ & $-*$ & - $^{*}$ \\
Gelatin/India ink & $100 \%$ & $55 \%$ & $100 \%$ & $100 \%$ & $30 \%$ \\
Saline/India ink & $100 \%$ & $100 \%$ & $100 \%$ & $-*$ & $40 \%$ \\
\hline
\end{tabular}

$\overline{A C P}$, Antegrade cerebral perfusion; $L S A$, left subclavian artery; $C C A$, common carotid arteries; $S C A$, subclavian arteries. *Experiment was not performed.

were occluded at their origins, and the descending aorta was occluded just distal to the origin of the left subclavian artery. The gelatin/India ink marker was directed into only the common carotid arteries (Figure 1,E). Perfusion with the saline/India ink marker was not performed.

In the last group (group VI), blood flow to the spinal cord via the vertebral circulation alone was studied in 1 rat by perfusing both subclavian arteries after both common carotid arteries were occluded at their origins. The descending aorta was also occluded just distal to the origin of the left subclavian artery. The gelatin/India ink marker was directed into both subclavian arteries alone (Figure $1, F$ ). Only 1 rat was used in this group inasmuch as the experimental setup had no clinical relevance. We wanted to see the territorial supply of the subclavian arteries to the spinal cord from an anatomic basis only. Perfusion with the saline/India ink marker was not performed.

\section{RESULTS}

Perfusion with colloidal carbon was more effective in depicting perfusion of the spinal vessels than E20 (Figure 2). Furthermore, colloidal carbon was easier to handle and left less contamination. As a result, colloidal carbon was our preferred choice of vascular marker.

In the control rats (group I), all vessels on the brain surface and spinal cord were filled completely using the colloidal carbon vascular marker mixed with either gelatin or saline (Figures 1, $A$, and 2).

In group II, where unilateral ACP was replicated via the brachiocephalic artery, the proximal $60 \%$ of the anterior

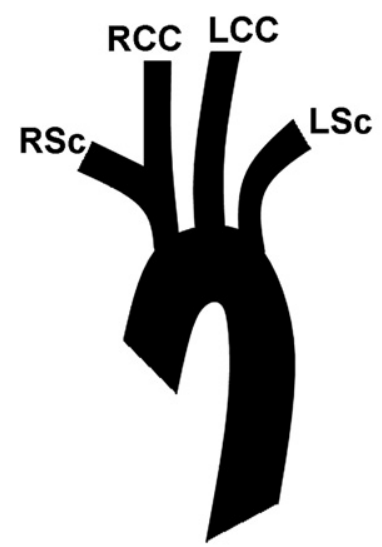

A

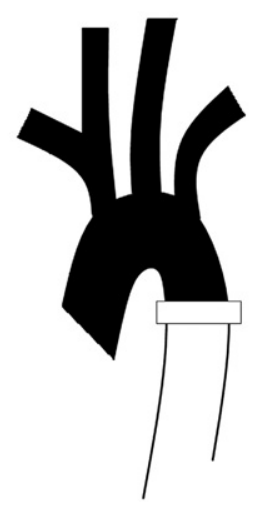

D

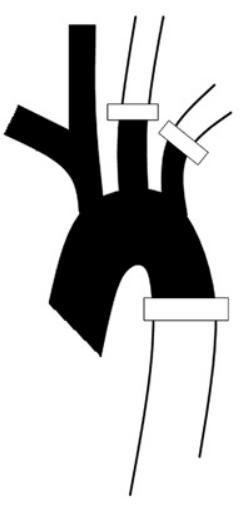

B

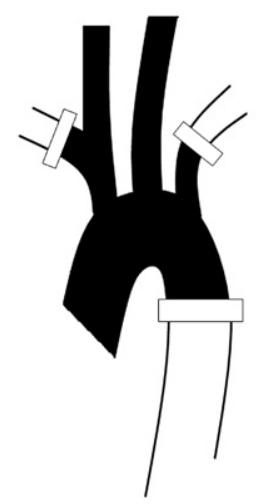

E

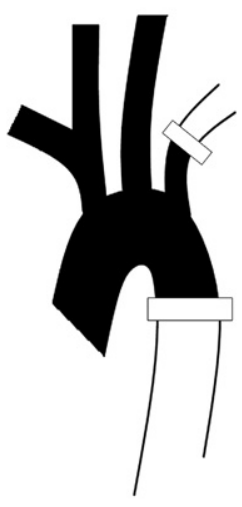

C

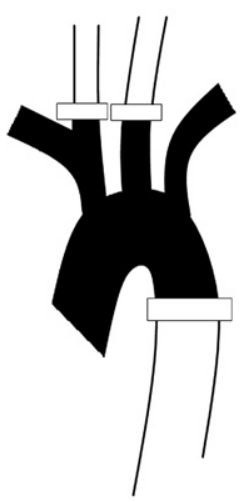

$\mathbf{F}$

FIGURE 1. Position of ligatures and vessels perfused. Position of ligatures indicated by white boxes. A, Control. B, Unilateral cerebral perfusion. C, Bilateral cerebral perfusion. D, Bilateral cerebral perfusion with additional left subclavian artery perfusion. E, Common carotid artery perfusion. F, Subclavian artery perfusion. $R S c$, Right subclavian artery; $R C C$, right common carotid artery; $L C C$, left common carotid artery; $L S c$, left subclavian artery. 

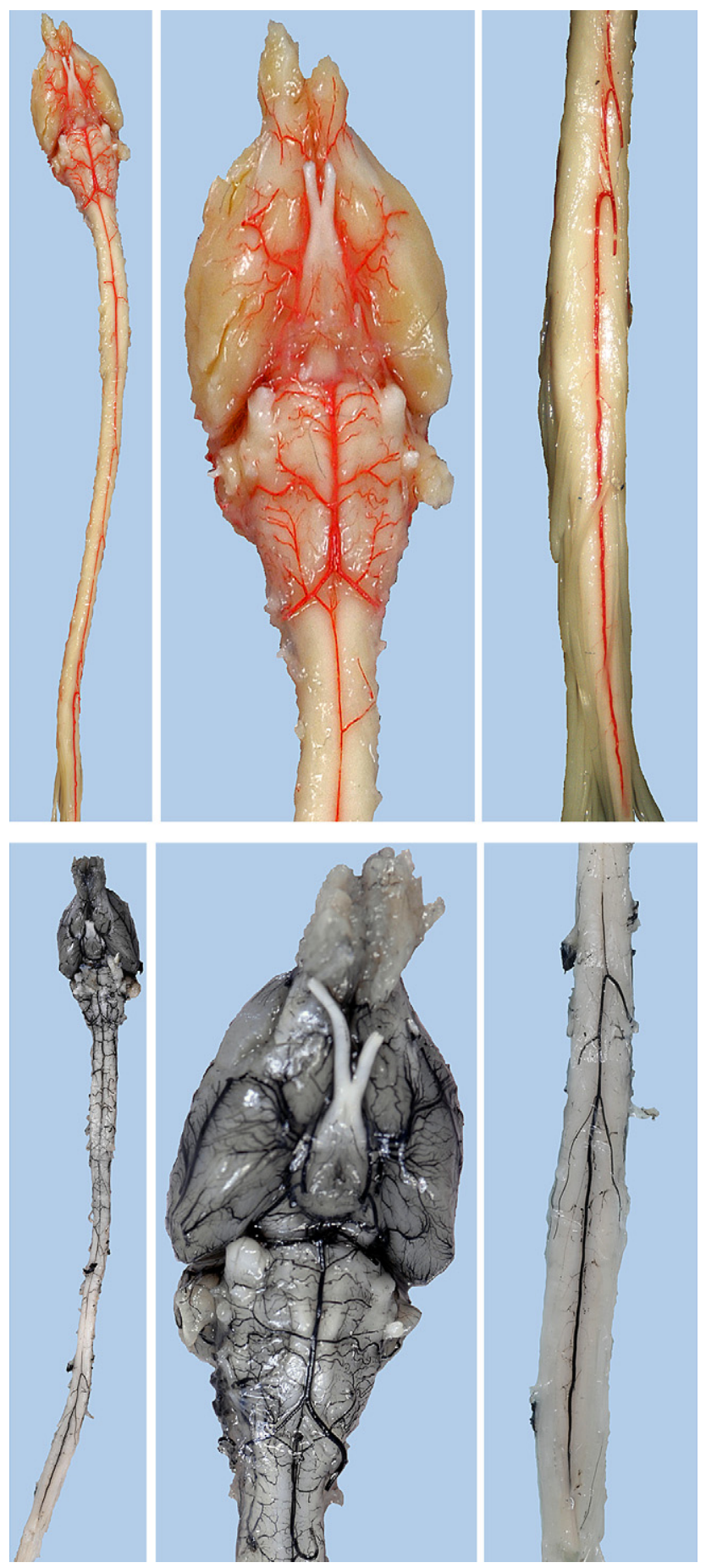

FIGURE 2. Group I: Control experiments showing the arterial filling in the brain and spinal cord. Top figures show perfusion using the E20 vascular marker. Botton figures show perfusion using the gelatin/India ink vascular marker.

spinal artery and the proximal $50 \%$ of the posterior spinal arteries were filled using the gelatin/India ink marker, whereas $100 \%$ filling of all vessels occurred in the rats perfused with saline/India ink (Figures 1, B, and 3). Despite proximal occlusion, both the left common carotid and left subclavian arteries filled in a retrograde direction. All cerebral, cerebellar, and vessels along the brainstem were filled. Distal to the aortic occlusion there was no downstream filling of the aorta or any of its major branches.

In group III, where bilateral ACP was replicated by injecting the brachiocephalic and left common carotid arteries, both the anterior and posterior spinal arteries filled completely along the length of the cord, down to the conus medullaris, using both colloidal carbon vascular markers (gelatin or saline) (Figure 1, C, and 4). All cerebral, cerebellar, and vessels along the brainstem were filled. The left subclavian artery filled in a retrograde direction despite the vessel being occluded at its origin. Distal to the aortic occlusion there was no downstream filling of the aorta or any of its major branches. Cross-sectional anatomy revealed that the penetration within the spinal cord was complete (Figure 5).

In group IV, where bilateral ACP was achieved in a similar fashion to group III but with the addition of perfusing the left subclavian artery, the anterior and posterior spinal arteries filled completely along the length of the cord using the gelatin/India ink marker (Figure 1,D). All cerebral, cerebellar, and vessels along the brainstem were filled.

Group V examined spinal cord perfusion alone with ACP via the common carotid arteries with occlusion of both left and right subclavian arteries. The gelatin/India ink marker flowed readily into the carotid system and then filled the vertebrobasilar system in a retrograde direction (Figure 1, $E$ ). Both subclavian arteries also filled in a retrograde fashion. Only the proximal $30 \%$ of the anterior and posterior spinal arteries were shown to be filled with the vascular marker (Figure 6, A). All cerebral, cerebellar, and vessels along the brainstem were filled. Distal to the aortic occlusion there was no downstream filling of the aorta or any of its major branches.

In group VI, perfusion was via the subclavian arteries alone with occlusion of both common carotid arteries, and spinal blood flow distribution was examined specifically (Figure 1,F). Both vertebral arteries filled and then perfused the cerebral and cerebellar vessels and the carotid vessels in a retrograde fashion. Only the proximal $40 \%$ of the anterior and posterior spinal arteries were shown to be filled with the vascular marker (Figure 6,B). In contrast to all other rat groups, filling of the cerebral and cerebellar vessels in group VI was poor and not to any meaningful extent. Only perfusion using the gelatin/India ink marker was performed. Perfusion using the saline/India ink marker was not undertaken in this group.

\section{DISCUSSION}

Our study examined the effectiveness of different approaches to ACP in maintaining blood flow to the brain and spinal cord. With respect to the brain, our study shows 


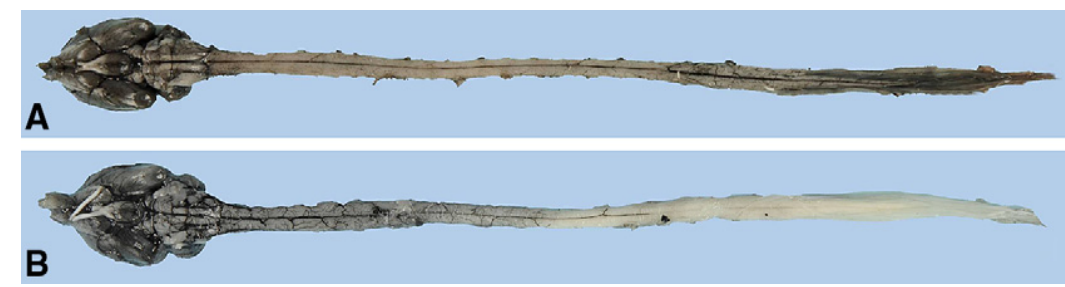

FIGURE 3. Group II: Replication of unilateral ACP. A, Perfusion using the gelatin/India ink marker. B, Perfusion using the saline/India ink marker.

that with either colloidal carbon marker, unilateral or bilateral ACP provides good cerebral and cerebellar perfusion under the experimental conditions used. With respect to the spinal cord, our study shows that with a less viscous marker (saline-based) full spinal cord perfusion was possible with unilateral or bilateral ACP. However, with a more viscous marker (gelatin-based) spinal cord perfusion was markedly reduced with unilateral ACP, whereas with bilateral ACP full spinal cord perfusion was evident. This suggests that in clinical contexts of any limitation of perfusion (whether by viscosity of blood at hypothermic state, or by pathology such as atheroma or dissected vessel) bilateral ACP may offer a safety advantage over unilateral ACP for spinal cord perfusion.

This may help to explain some of the findings of Malvindi, Scrascia, and Vitale ${ }^{9}$ in their meta-analysis of 17 studies when they concluded that both unilateral and bilateral techniques were acceptable but suggested that if the selective ACP time is expected to be greater than 40 to 50 minutes, then bilateral ACP was the safest technique.

How the spinal cord is perfused under these conditions of circulatory arrest with ACP is not fully understood. Although bilateral ACP may be the preferred technique on a theoretical basis for maintaining cerebral and spinal perfusion during hypothermic circulatory arrest, unilateral cannulation is still performed for some surgical procedures. Although the incidence of neurologic deficits after surgery is low with both techniques, complications that include paraplegia and paraparesis are devastating. ${ }^{9}$

To examine the impact of different approaches to ACP on spinal perfusion, we used a rat model because of its similarity to human spinal vasculature. ${ }^{13,14}$ We found that the most effective perfusion of the spinal cord was achieved by perfusing both the brachiocephalic and left common carotid arteries with or without additional left subclavian artery perfusion. This situation represents bilateral cerebral perfusion, which is the most widely used technique for maintaining cerebral perfusion. With bilateral ACP, the entire length of the anterior and posterior spinal arteries and the penetrating arteries of the spinal neuropil filled well with both colloidal carbon vascular markers. Unilateral ACP established via cannulation of the brachiocephalic artery alone was less effective, with the anterior and posterior spinal arteries filling only to the level of the lower thoracic vertebrae with the gelatin/India ink marker.

Our study also examined the relative importance of bilateral common carotid artery perfusion alone compared with bilateral subclavian artery perfusion alone in providing spinal cord blood supply. Using the more viscous marker, we showed that there are significant limitations in spinal cord blood flow if only both the common carotid arteries are used or if the subclavian arteries alone are injected. In our study, direct perfusion of the common carotid arteries alone resulted in perfusion of only the proximal $30 \%$ of the spinal cord by way of retrograde flow through the vertebrobasilar system, with extensive cerebral and cerebellar perfusion. On the other hand, perfusion of the subclavian arteries alone resulted in greater spinal cord perfusion (up to $40 \%$ ) at the prohibitive cost of near complete absence of perfusion to the cerebral cortex and cerebellum. Thus ACP with contribution from the common carotid arteries and at least one of the subclavian arteries provides much better perfusion both to the spinal cord and to the brain.

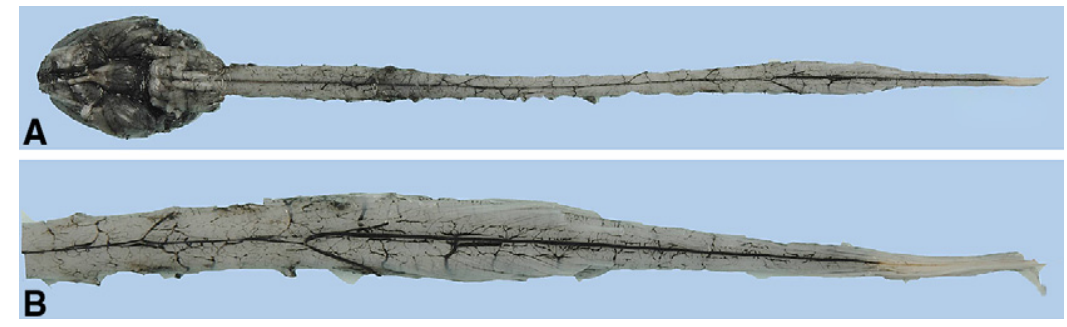

FIGURE 4. Group III: Replication of bilateral ACP. A, Ventral view of brain and spinal cord showing maximal filling. B, A higher magnification of the lower spinal cord shown in Figure 4, A. 


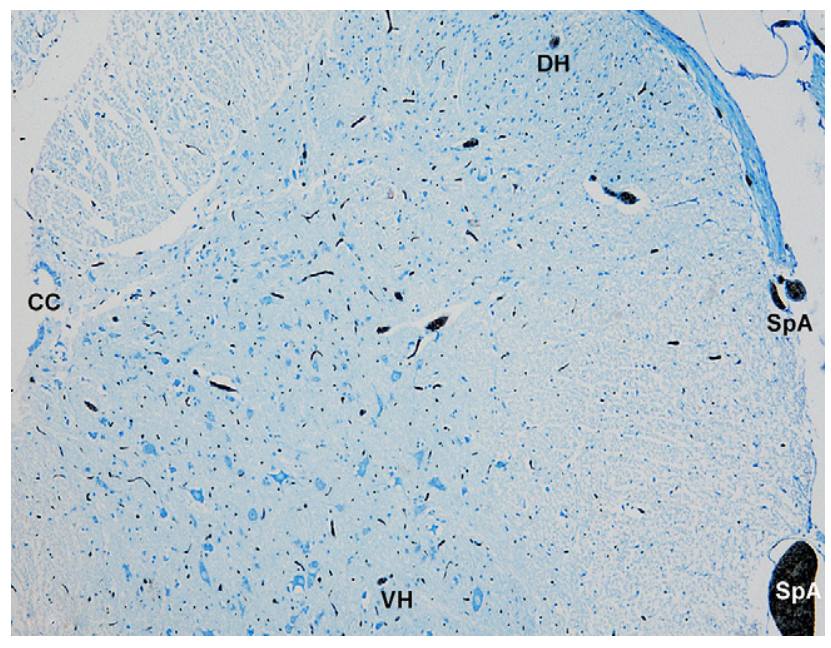

FIGURE 5. Horizontal section of the thoracic spinal cord stained with methyl green showing filling of the microvasculature in the gray and white matter with colloidal carbon. $C C$, Central spinal canal; $D H$, dorsal horn; $V H$, ventral horn; $S p A$, branch of the spinal artery on the surface of the cord.

The left subclavian artery is sometimes perfused as an adjunct to bilateral ACP, depending on the surgeon's preference. ${ }^{16,17}$ In rats in which the left subclavian artery was perfused as an adjunct to bilateral ACP (group IV), complete filling of the spinal arteries was also achieved, with no compromise to cerebral or cerebellar supply. When bilateral ACP was performed using the gelatin/India ink marker without injecting the left subclavian artery directly (group III), the left subclavian artery and its distal branches were still filled by retrograde flow through the left vertebral artery, and complete distribution of perfusate was seen in the spinal cord. Our results, therefore, suggest that cannulation of the left subclavian artery may be redundant in the surgical context with one important caveat, namely, that in situations of vascular compromise, it may be valuable to perfuse the left subclavian artery. Vascular compromise could include occlusion of the right vertebral artery, a dominant left vertebral artery, carotid artery disease, or inadequate intracranial arterial communication. ${ }^{3,7}$
Clinical studies vary in their clinical recommendations. Some claim that unilateral ACP can be as effective as bilateral cerebral ACP for maintaining perfusion to the brain in some clinical situations. ${ }^{9}$ Unilateral cerebral perfusion is often used clinically in patients undergoing hemiarch replacement for acute type A aortic dissection. ${ }^{7}$ Immer and associates ${ }^{18}$ found that unilateral antegrade perfusion allowed better early survival than bilateral perfusion and caused less postoperative permanent neurologic deficit. The difference between the clinical findings of unilateral cerebral perfusion and our study may be due to differences in the perfusion technique that were used as well as anatomic differences between the rat and the human clinical scenario, which includes pathologic conditions such as atheroma or vessel dissection on occasion.

Although our use of a rat model has limitations, such a study would be impossible in humans. There are a number of limitations and differences. Our vascular perfusion technique also differs from the clinical situation in which anticoagulated blood is infused continuously into the vasculature, with visceral circulatory arrest maintained at specific temperatures. Anticoagulation probably enables greater filling of vessels than what could be achieved with the technique used in this study. Furthermore, in the rats that were studied, anastomoses between branches of the internal thoracic artery and the posterior intercostal arteries were severed to expose the thoracic cavity. Filling of the descending aorta through this route was therefore prevented. Under surgical conditions, arterial supply to the descending aorta is likely to be assisted by these vessels. This system may facilitate perfusion of segmental medullary and radicular arteries that arise from segmental branches of the aorta, aiding the supply of the spinal arteries distally. ${ }^{13,19,20}$ Finally, we used the degree of vessel filling as a proxy measure for the extent of spinal cord tissue penetration. The degree of spinal cord tissue perfusion was only assessed by histologic examination at 3 levels: midcervical, midthoracic, and midlumbar; thus entire perfusion could not be ascertained unless serial sections were performed.

Many textbooks stress the importance of watershed areas and major areas of spinal artery perfusion reinforcement, in

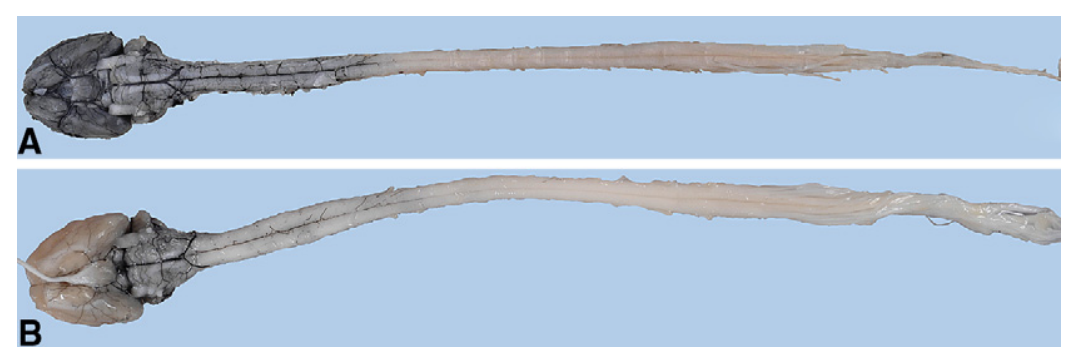

FIGURE 6. A, Group V: Perfusion of common carotid arteries alone with gelatin/India ink marker. Only proximal 30\% of spinal cord arteries are filled. Good cerebral filling. B, Group VI: Perfusion of both subclavian arteries alone with gelatin/India ink marker. Only proximal $40 \%$ of spinal cord arteries are filled. Poor cerebral filling. 
particular the artery of Adamkiewicz in the thoracolumbar region. The implication is that a "top down" approach that uses only the avenues generated by ACP would create risk to the spinal cord neurones, particularly the distal segments. Our study found this not to be the case, inasmuch as extensive perfusion of the spinal cord neuropil was demonstrated by histologic study at all levels of the cord using bilateral ACP with all the markers we used, and with unilateral ACP using a less viscous marker (saline/India ink). Supply of the spinal nutrient arteries has long been thought to arise from 3 longitudinal spinal arteries, but this study and several others have demonstrated that a collateral network of vessels is responsible for the supply of the spinal cord. Inputs into this network include not only the segmental vessels such as the artery of Adamkiewicz, but also the subclavian arteries and its branches. ${ }^{21}$ This study found that sufficient perfusion of the input vessels proximally was enough to ensure supply to the spinal cord neurons distally without the need for segmental vessel perfusion.

In conclusion, we have shown, at least in rats, that brain perfusion is preserved with both unilateral ACP (brachiocephalic artery) and bilateral ACP (brachiocephalic artery and left common carotid artery, with or without left subclavian artery). However, with different approaches to ACP, there is variation in anatomically defined perfusion of the spinal cord under conditions that simulate circulatory arrest and ACP. For spinal cord perfusion under simulated circulatory arrest, ACP techniques that use the brachiocephalic artery alone, the common carotid arteries alone, or the subclavian arteries alone are less effective than techniques that use both the brachiocephalic and the left common carotid arteries together. In situations in which there are known limitations to perfusion of the brachiocephalic or the left common carotid artery or the circle of Willis, additional perfusion of the left subclavian artery may be beneficial. Alternatively, deeper and more protective hypothermia may be indicated.

We thank Dr Beau Pontré for radiography, Dr Phil Blyth for computing, and Dr Satya Amirapu for assistance with histology. The skills of Mr Nick Duggan in photography were greatly appreciated.

\section{References \\ 1. Baumgartner FJ, Omari B. Extracorporeal techniques during graft replacement of suprarenal abdominal aortic dissections. Panminerva Med. 1998;40:350.}

2. Hagl C, Khaladj N, Karck M, Kallenbach K, Leyh R, Winterhalter M, et al. Hypothermic circulatory arrest during ascending and aortic arch surgery: the theoretical impact of different cerebral perfusion techniques and other methods of cerebral protection. Eur J Cardiothorac Surg. 2003;24:371-8.

3. Apostolakis E, Akinosoglou K. The methodologies of hypothermic circulatory arrest and of antegrade and retrograde cerebral perfusion for aortic arch surgery. Ann Thorac Cardiovasc Surg. 2008;14:138-48.

4. Kouchoukos NT, Masetti P, Rokkas CK, Murphy SF. Hypothermic cardiopulmonary bypass and circulatory arrest for operations on the descending thoracic and thoracoabdominal aorta. Ann Thorac Surg. 2002;74:S1885-7; discussion S92-8.

5. Frist WH, Baldwin JC, Starnes VA, Stinson EB, Oyer PE, Miller DC, et al. A reconsideration of cerebral perfusion in aortic arch replacement. Ann Thorac Surg. 1986;42:273-81.

6. Kazui T, Washiyama N, Muhammad BA, Terada H, Yamashita K, Takinami M, et al. Total arch replacement using aortic arch branched grafts with the aid of antegrade selective cerebral perfusion. Ann Thorac Surg. 2000;70:3-8; discussion 9.

7. Kazui T, Yamashita K, Washiyama N, Terada H, Bashar AH, Suzuki K, et al. Aortic arch replacement using selective cerebral perfusion. Ann Thorac Surg. 2007;83:S796-8; discussion S824-31.

8. Khaladj N, Shrestha M, Meck S, Peterss S, Kamiya H, Kallenbach K, et al. Hypothermic circulatory arrest with selective antegrade cerebral perfusion in ascending aortic and aortic arch surgery: a risk factor analysis for adverse outcome in 501 patients. J Thorac Cardiovasc Surg. 2008;135:908-14.

9. Malvindi PG, Scrascia G, Vitale N. Is unilateral antegrade cerebral perfusion equivalent to bilateral cerebral perfusion for patients undergoing aortic arch surgery? Interact Cardiovasc Thorac Surg. 2008;7:891-7.

10. Byrne JG, Fitzgerald DJ, Aranki SF. Simultaneous selective cerebral perfusion and systemic circulatory arrest through the right axillary artery for aortic surgery. J Card Surg. 1998;13:236-8.

11. Sanioglu S, Sokullu O, Ozay B, Gullu AU, Sargin M, Albeyoglu S, et al. Safety of unilateral antegrade cerebral perfusion at 22 degrees $\mathrm{C}$ systemic hypothermia. Heart Surg Forum. 2008;11:E184-7.

12. Olsson C, Thelin S. Antegrade cerebral perfusion with a simplified technique: unilateral versus bilateral perfusion. Ann Thorac Surg. 2006;81:868-74.

13. Brockstein B, Johns L, Gewertz BL. Blood supply to the spinal cord: anatomic and physiologic correlations. Ann Vasc Surg. 1994;8:394-9.

14. Greene E. Anatomy of the rat. New York: Hafner Pub. Co; 1968.

15. Piasecki C, Wyatt C. Patterns of blood supply to the gastric mucosa. A comparative study revealing an end-artery model. J Anat. 1986;149:21-39.

16. Svensson LG, Nadolny EM, Penney DL, Jacobson J, Kimmel WA, Entrup MH, et al. Prospective randomized neurocognitive and S-100 study of hypothermic circulatory arrest, retrograde brain perfusion, and antegrade brain perfusion for aortic arch operations. Ann Thorac Surg. 2001;71:1905-12.

17. Ueda T, Shimizu H, Ito T, Kashima I, Hashizume K, Iino Y, et al. Cerebral complications associated with selective perfusion of the arch vessels. Ann Thorac Surg. 2000;70:1472-7.

18. Immer FF, Moser B, Krahenbuhl ES, Englberger L, Stalder M, Eckstein FS, et al. Arterial access through the right subclavian artery in surgery of the aortic arch improves neurologic outcome and mid-term quality of life. Ann Thorac Surg. 2008;85:1614-8; discussion 1618.

19. Greathouse DG, Halle JS, Dalley AF 2nd. Blood supply to the spinal cord. Phys Ther. 2001;81:1264-5.

20. Tveten L. Spinal cord vascularity. II. Extraspinal sources of spinal cord arteries in the rat. Acta Radiol Diagn (Stockh). 1976;17:167-79.

21. Griepp RB, Griepp EB. Spinal cord perfusion and protection during descending thoracic and thoracoabdominal aortic surgery: the collateral network concept. Ann Thorac Surg. 2007;83:S865-9; discussion S90-2. 